\title{
FACTORS AFFECTING PRECISION AGRICULTURE ADOPTION: A SYSTEMATIC LITTERATURE REVIEW
}

\author{
Taoufik YATRIBI ${ }^{1}$
}

date of paper receipt:

17.08.2020.

Review Article date of sending to review:

25.08.2020.

doi: 10.2478/eoik-2020-0013 date of review receipt:

09.09.2020.

UDK 631.153:061.61]:352.07

${ }^{1}$ Department of Rural Economy, National School of Agriculture in Meknès, Morocco

\begin{abstract}
The aim of this paper is to present the main advances in the adoption of precision agriculture technologies. While we are witnessing the emergence of a literature dedicated to the adoption of new technologies, this theme still suffers from a lack of consensus on its conceptualization. Based on the prisma statement method (Preferred Reporting Items for Systematic Reviews and Meta-Analyzes), the objective is to carry out a review of the systemic literature in order to identify the main factors of adoption of the technologies of precision agriculture over the past ten years. The results show that individual factors are the most empirically identified as determining factors in the adoption of precision agriculture technologies. That said, the farmer is at the center of the adoption decision. Perceived utility is the factor most identified in the literature as the determinant of adoption.
\end{abstract}

Keywords:

Technology adoption, technological innovations, precision agriculture, Systematic reviews

JEL: R11 


\section{INTRODUCTION}

The introduction of technological innovations has now become an imperative. The modernization of the economic sectors is more and more necessary, in particular that there are economic, social, and environmental issues. This latest health crisis (COVID-19) has reinforced this need to move to a digital economy, especially when this pandemic has paralyzed many sectors, thereby threatening food security. The agricultural sector is then one of the sectors most concerned. Indeed, although this sector is certainly more innovative than in the 80 s and 90s, it remains insufficiently modernized to meet current challenges (Food security, problems of productivity and competitiveness, jobs, reduction of social inequalities...). Empirical studies suggest that new technologies have positive agronomic, economic and environmental effects (Koutsos and Menexes, 2019). Experts agree today that technological innovation is an asset to gain time and precision for the benefit of the farmer, the farm and the environment.

At this level, precision agriculture (PA) appears to be a major asset in making agriculture an innovative and responsible sector. The literature indicates that PA technologies have one thing in common: optimizing agricultural production. PA technologies today allow farmers to apply the right dose of input at the right time, reduce the use of phytosanitary products, predict the appearance of diseases in a plot, reduce energy consumption fossil or the arduousness of the work. It also makes it possible to precisely determine the water, fertilizer and phytosanitary product requirements for crops. It thus becomes possible to optimize the use of chemical inputs and equipment, as well as a reduction in $\mathrm{C} 02$ emissions.

The adoption of PA technologies by farmers is the starting point for the development of innovative agriculture, in which farmers are the actors, and technologies are the tools for this development. Adoption itself is a complex part at this level. Previous studies show that the adoption of technology depends on many factors (socio-demographic, economic, institutional, etc.), and the farmer is at the center of the decision to adopt and accept the technology. Empirical studies suggest that the adoption decision would be shaped by several determining factors. From this perspective, questions arise: What actually depends on the adoption of PA technologies by the farmer? What can depend on attitude and his adoption decision? How can we explain the mechanisms of adoption of technologies by the farmer?

The aim of this article is to conduct a review of the systemic literature with the aim of identifying the main factors for the adoption of PA technologies on a global scale. Although the literature is abundant on this subject, there is however lack of works which synthesize research on this subject. Our goal is to take stock of research over the past ten years, with the aim of identifying the determining factors, their frequencies and their importance, but also to highlight the theoretical gaps, that is, to highlight what has not been covered in this research stream. The interest of this research lies in the understanding of the factors influencing the adoption of new technologies which are essential both for the decision-makers, the diffusers of these technologies (companies), and for the researchers who study the determinants of growth. Finally, interest in digitalization and the transition to a digital economy has only increased in recent years. This interest has just been reinforced by the COVID-19 pandemic. Public authorities therefore need to understand the adoption mechanisms in the farmer in order to be able to put in place a strategy for the development of digital agriculture. 


\section{MATERIALS AND METHODS}

This study is a systematic review of the literature, with the aim of researching and synthesizing scientific work on the determinants of the adoption of PA technologies. We opted for the prisma statement method (Preferred Reporting Items for Systematic reviews and Meta-Analyzes), which can be summarized in four steps: Identification - Selection - Eligibility - Inclusion. This systematic review of the literature covers the period 2010-2019, i.e. ten years of research. To identify research work, we targeted two databases: SCOPUS, and Science Direct, which are relevant and complementary search engines. To identify the publications, we did a search by keywords, at the level of titles and/or summaries. A transversal reading of the literature allowed us to identify these keywords. The table below presents the keywords used for this search :

Table 1. Keywords used in research

\begin{tabular}{|c|c|}
\hline & Keywords \\
\hline Group 1 & $\begin{array}{c}\text { Precision agriculture, Technology Adoption, precision farming, Diffusion } \\
\text { of innovation, Innovation in Agriculture, Agricultural practice, Intensity of } \\
\text { adoption }\end{array}$ \\
\hline Group 2 & $\begin{array}{c}\text { Variable rate technology, GPS guidance, GPS autosteer, Remote sensing, } \\
\text { Connected sensors, Agricultural robots, agriculture drone, Variable rate } \\
\text { technology, variable rate fertiliser, High-tech Agriculture, Unmanned Aircraft } \\
\text { Systems, }\end{array}$ \\
\hline
\end{tabular}

For selection, the studies had to meet the following inclusion and exclusion criteria: First, only articles review were selected for this study. The articles were to be published in English or French between January 2010 and December 2019. The subject covered was to focus specifically on the determinants of the adoption of PA technologies. The exclusion criteria were non-response to the inclusion criteria, in other words, articles published before January 2010 are not included, and also articles published in languages other than English and French, articles off topic, documents other than journal articles and journal articles, and finally duplicate articles.

The article admissibility review was conducted by the author of this work. The quality of the selected articles was then assessed. A quality score of $60 \%$ had to be reached for an item to be selected. Finally, two software programs were used in this work. The Zotero tool was used first to import and filter the articles, then we exported the selected articles to the Nvivo software to analyze content.

\section{RESULTS}

In what follows, we will successively present the selection procedure, the main results from the synthesis of this literature on the determinants of the adoption of PA technologies. We then discuss the determining factors of adoption, their frequency of identification, their importance. Previously, we present some indications on the selected articles.

\subsection{STUDY SELECTION}

An initial bibliographic search using the keyword "Precision agriculture" in the two databases (Scopus and Science Direct) gave rise to 12,117 publications. The search equation (Eq1) and the filter used made it possible to identify 221 articles (106 in the Scopus database, and 115 in the Science Direct database). These 221 articles were exported to the Zotero software for initial processing. A vertical reading made it possible to delete 43 double articles. Then, we tested the eligibility of the articles by reading the titles and the summaries to check if the articles correspond 
to the objective of this study. This step removed 97 articles that are not specifically concerned with identifying the determinants of the adoption of precision farming technologies. We point out that we have targeted empirical work and work with qualitative approaches. The qualitative approach in research has become a trend in recent years. Researchers tend to favor the qualitative approach in order to better contextualize their work. Finally, the full-text analysis saved 41 articles for this systematic literature review.

Figure 1. Flow chart of the bibliographic research

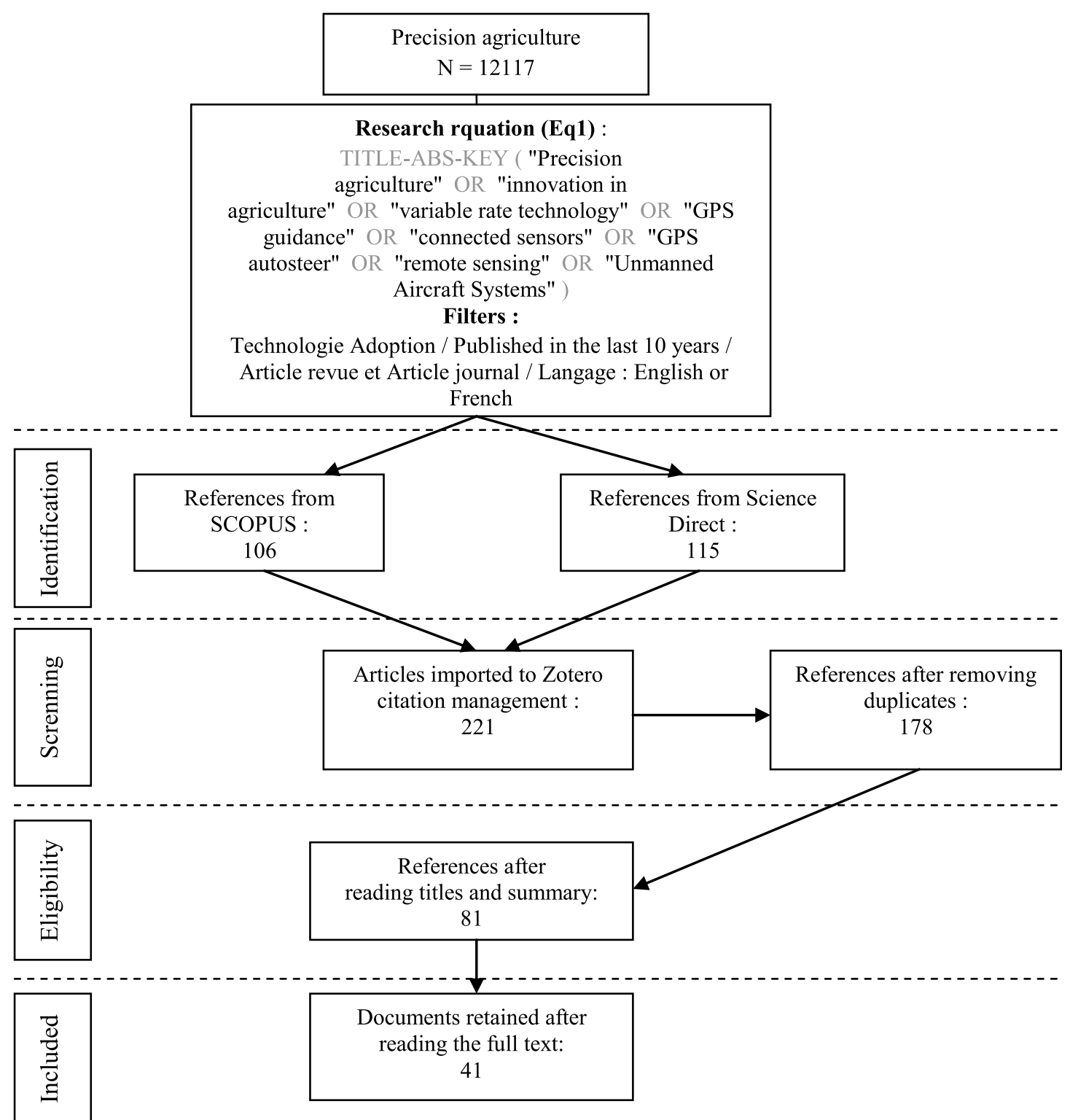




\subsection{GENERAL INFORMATION ON SELECTED ARTICLES}

The final sample consists of 41 articles written in English and published in English-language journals. About 60\% of the articles were published between 2017 and 2019, and 40\% were published in 2019. This shows that there is a recent dynamism in this field.

Figure 2. Number of publications per year

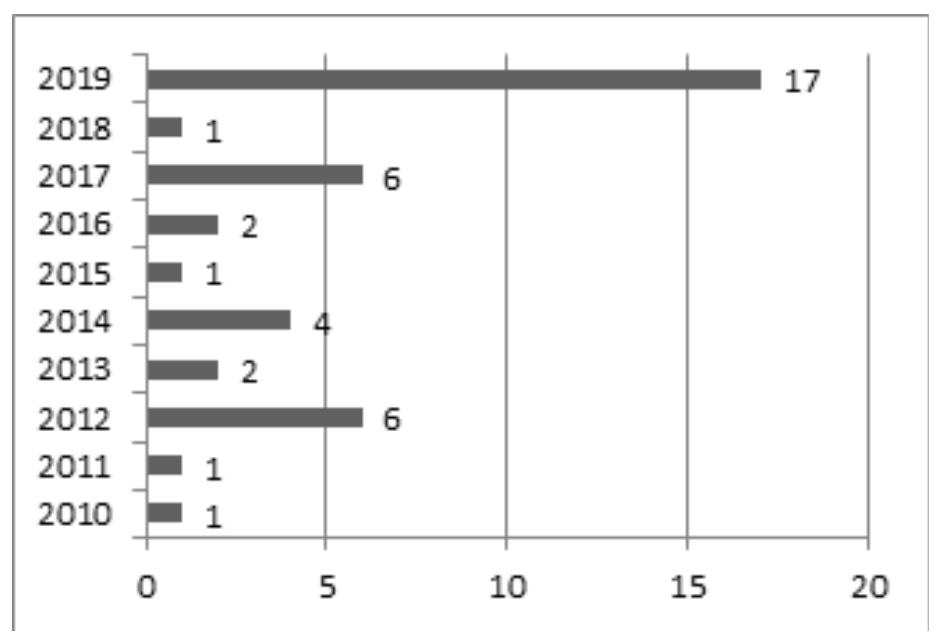

The sample of selected articles is heterogeneous and covers several continents. All geographic areas are represented but in varying proportions. This criterion was not taken into account in the inclusion criteria, we did not seek to have a balanced distribution. The quality and relevance of the articles were our priority in the choice. In terms of the breakdown of articles by country, the United-States leads with seven articles, or around $17 \%$ of the sample. It is followed by New-Zealand with 4 articles, approximately 9\% of the sample, and the United-Kingdom and Germany with 3 articles each. Less than $10 \%$ of the articles come from Africa, represented with 4 articles. The rest of the continent, Asia has eight articles in this literature review. The following graphic presents more details on the origin of the other papers. We will now focus on the theoretical frameworks of these publications

Figure 3. Number of publications by country

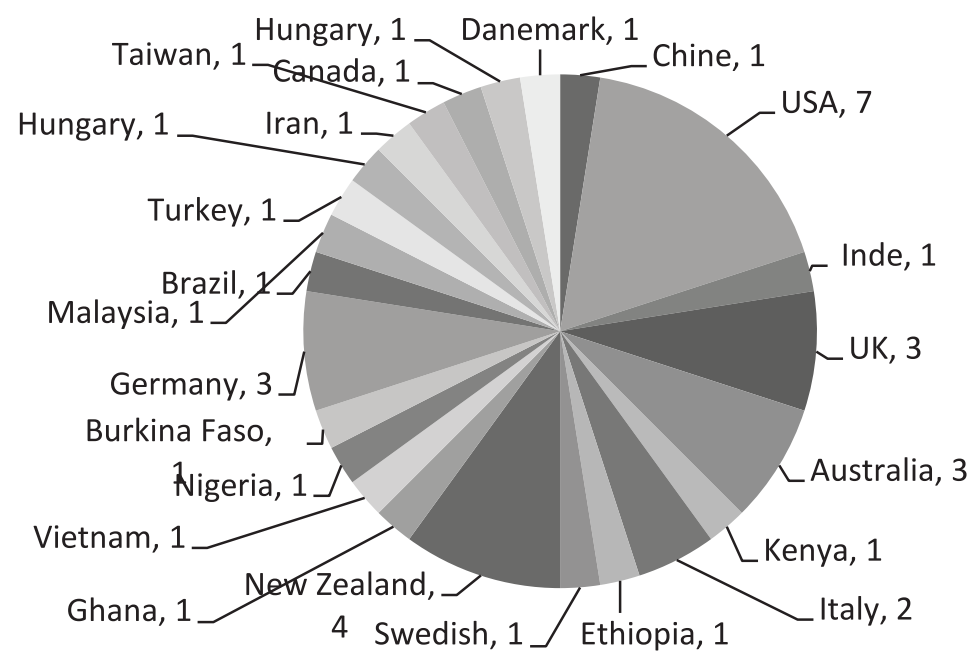




\section{FACTORS INFLUENCING THE ADOPTION OF PRECISION AGRICULTURE TECHNOLOGIES :}

Analysis of the literature revealed 21 variables that could influence the adoption of PA technologies. To give a broad overview of the research work, we offer in the table below, a summary of all the articles selected (Table 2).

We then grouped these variables into four groups of explanatory variables: individual factors, organizational factors, environmental factors and technological factors (Table 3). Thus, 10 individual factors were identified, or approximately $48 \%$ of all factors, with the highest frequency of identification by the researchers. The organizational and institutional factors are 4 factors each, or $19 \%$ for each of all the factors identified. Finally, the factors relating to the technology itself are identified as determinants of adoption, i.e. 3 factors identified.

Then, we can classify these determinants according to their degree of importance and influence on the adoption of new PA technologies. The following table shows the ranking of factors, specifying the number of times identified in the 41 articles consulted as determining factors for the adoption of PA technologies. 
Table 2. General summary of selected articles

\begin{tabular}{|c|c|c|c|c|c|c|c|c|c|c|c|}
\hline 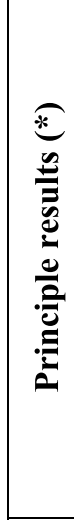 & 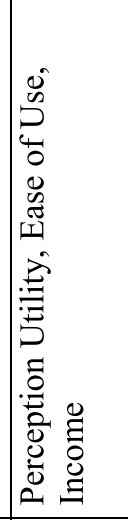 & 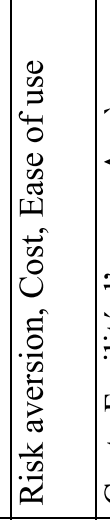 & 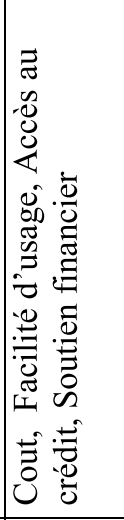 & 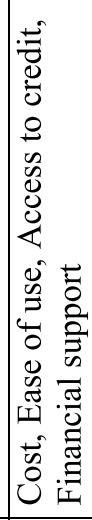 & 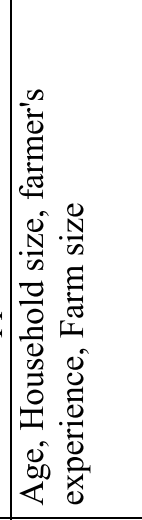 & 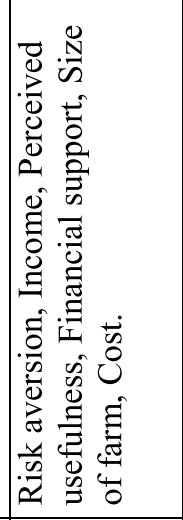 & 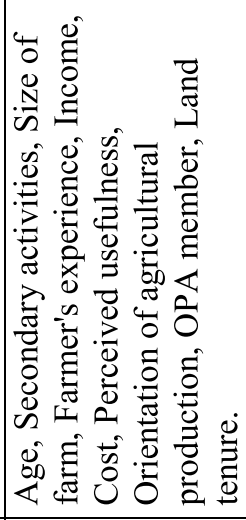 & 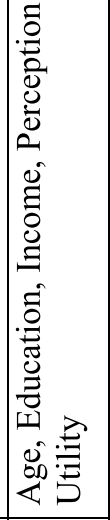 & 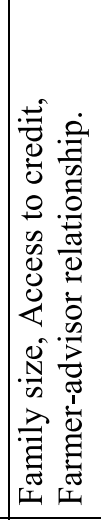 & 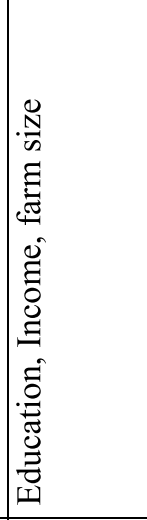 & 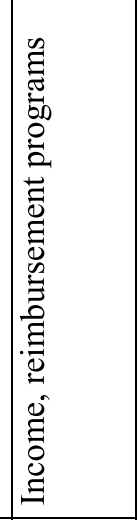 \\
\hline 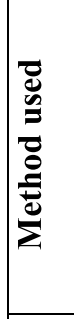 & 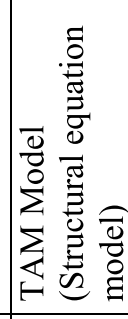 & 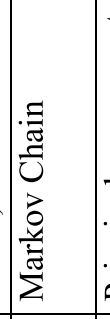 & 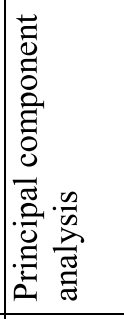 & 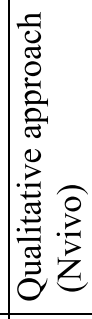 & 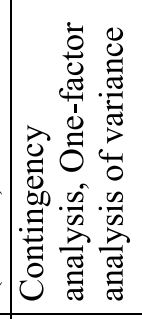 & 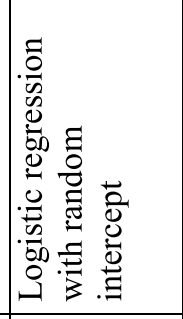 & 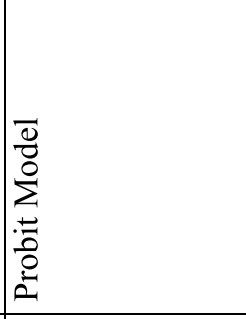 & 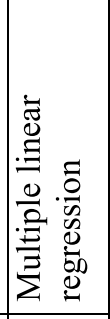 & 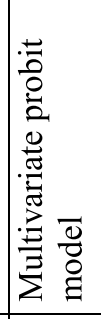 & 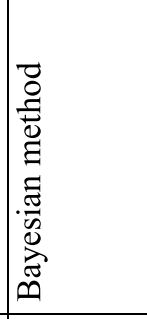 & 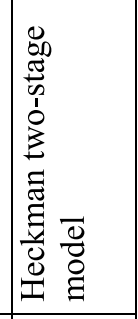 \\
\hline 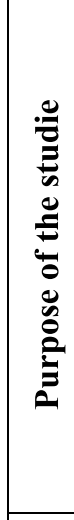 & 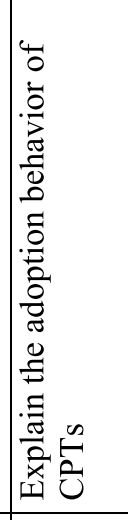 & 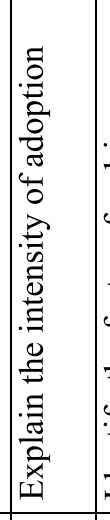 & 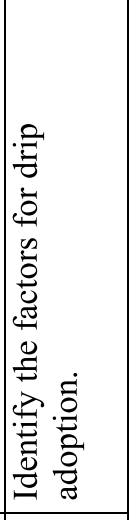 & 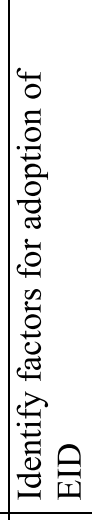 & 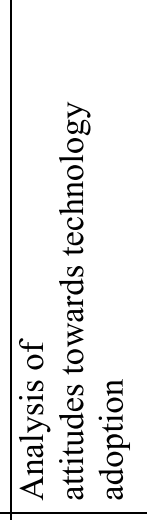 & 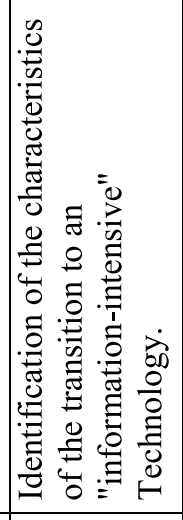 & 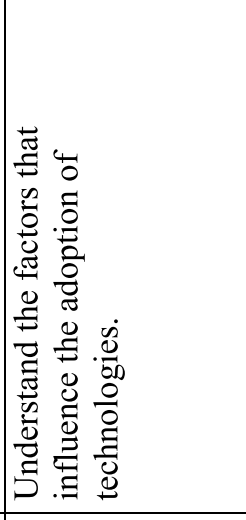 & 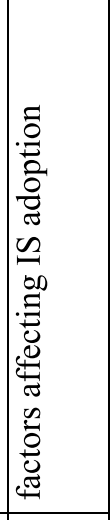 & 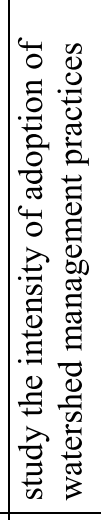 & 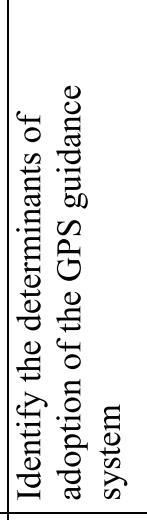 & 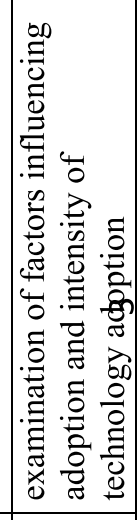 \\
\hline 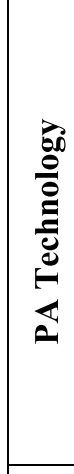 & 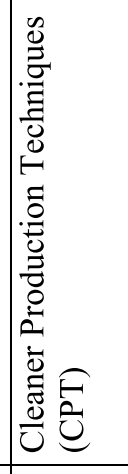 & 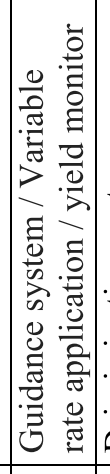 & 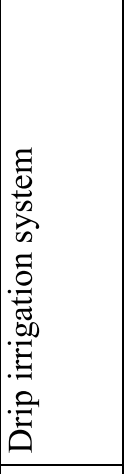 & 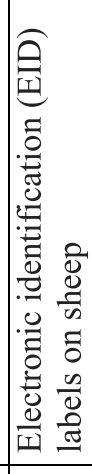 & 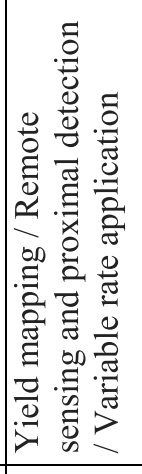 & 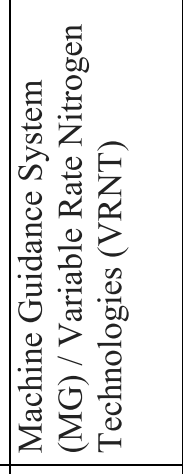 & 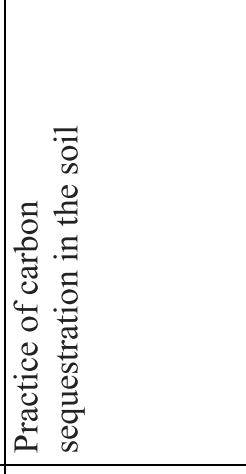 & 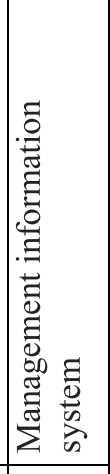 & 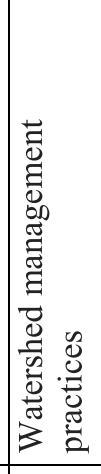 & 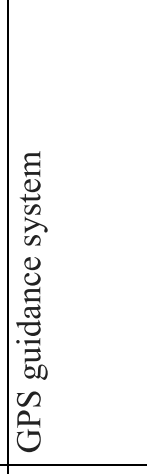 & 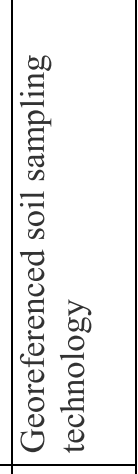 \\
\hline 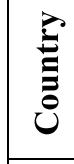 & ]ี & 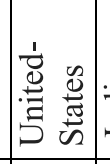 & 承 & 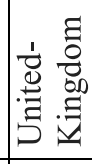 & 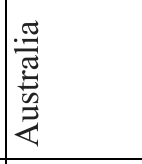 & $\begin{array}{l}\text { 咅 } \\
\text { 总 } \\
\text { 品 }\end{array}$ & 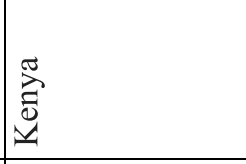 & 产 & 童 & 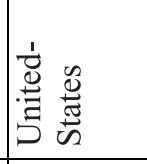 & 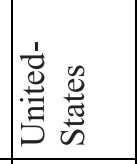 \\
\hline ¿્ & $\overrightarrow{\mathrm{i}}$ & 亏े & ᄅे & $\vec{i}$ & $\overrightarrow{\tilde{i}}$ & ᄅे & $\overrightarrow{\tilde{i}}$ & $\overrightarrow{\hat{i}}$ & ᄅे & $\overrightarrow{\tilde{i}}$ & $\stackrel{\infty}{\stackrel{\sim}{\tilde{N}}}$ \\
\hline 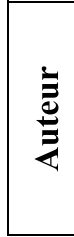 & 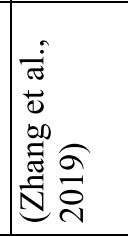 & 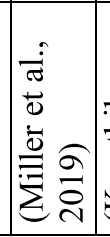 & 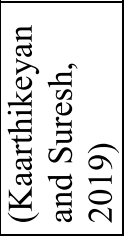 & 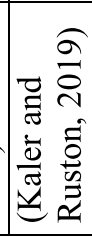 & 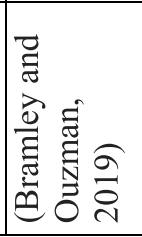 & 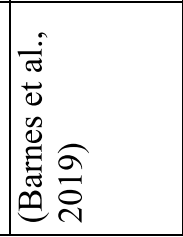 & 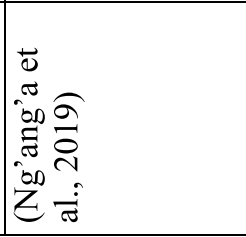 & 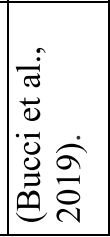 & 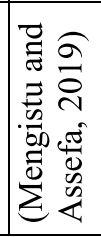 & 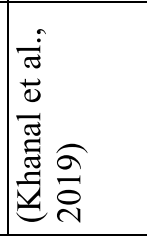 & 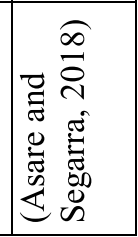 \\
\hline
\end{tabular}




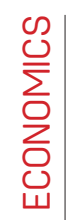

\begin{tabular}{|c|c|c|c|c|c|c|c|c|c|c|}
\hline 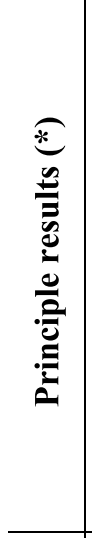 & 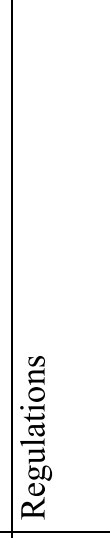 & 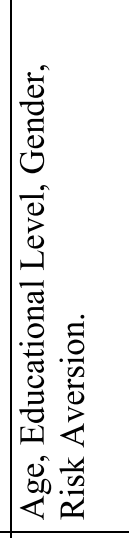 & 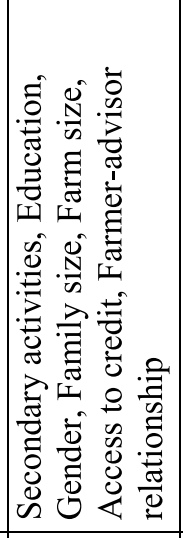 & 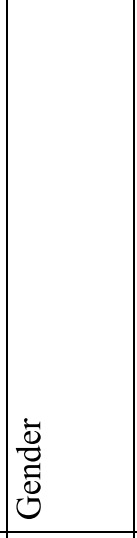 & 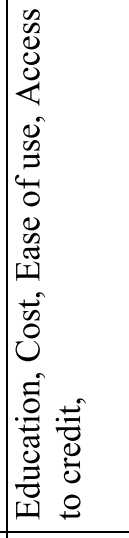 & 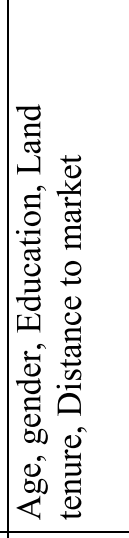 & 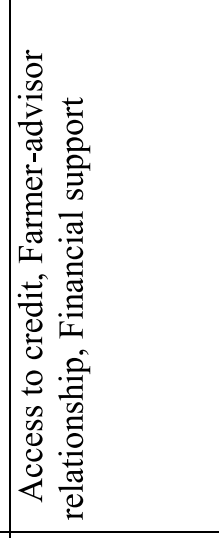 & 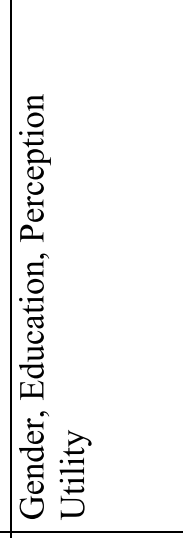 & 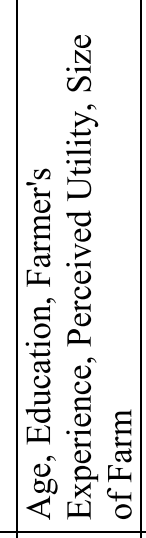 & 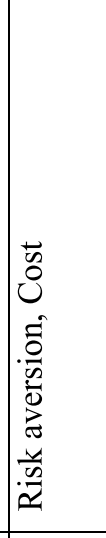 \\
\hline 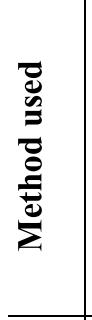 & 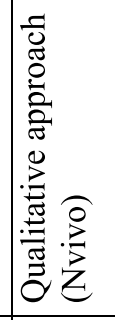 & 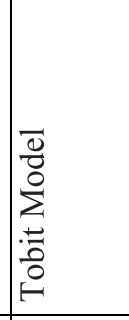 & 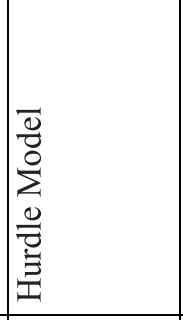 & 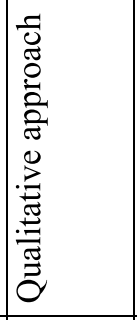 & 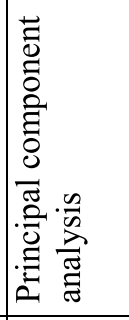 & 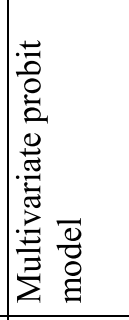 & 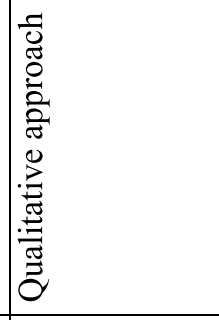 & 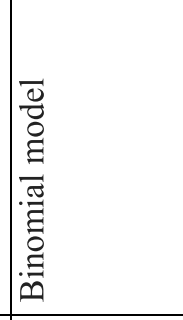 & 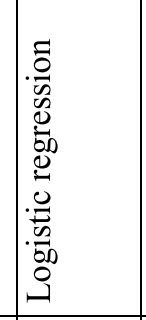 & 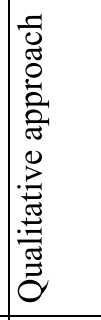 \\
\hline 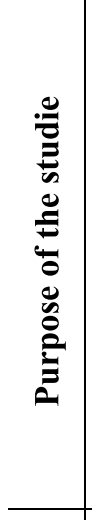 & 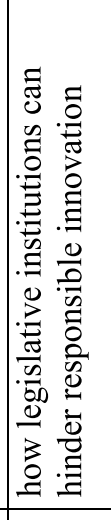 & 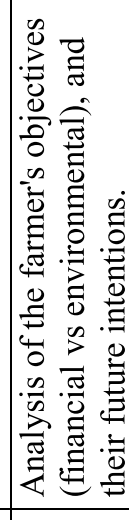 & 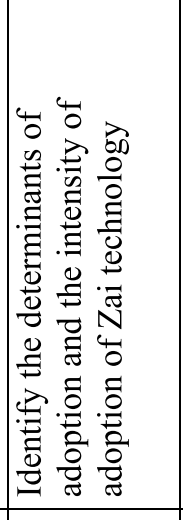 & 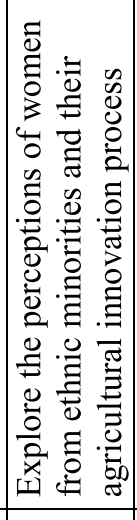 & 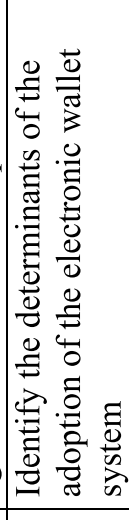 & 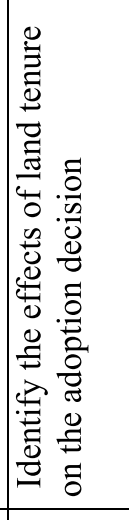 & 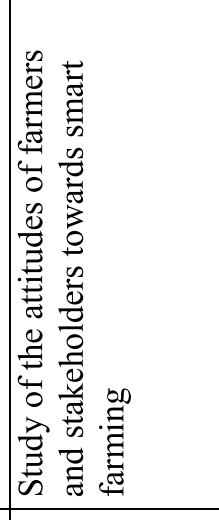 & 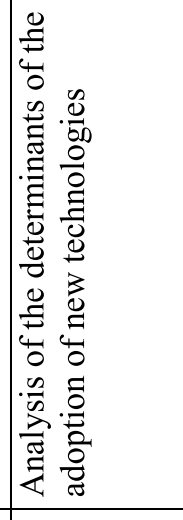 & 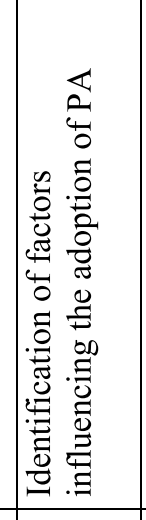 & 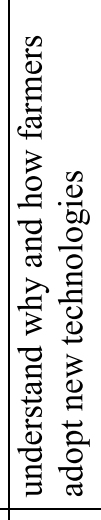 \\
\hline 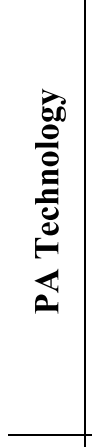 & 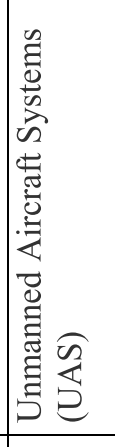 & 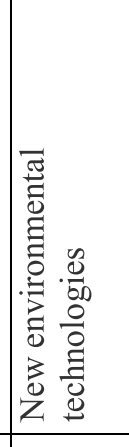 & 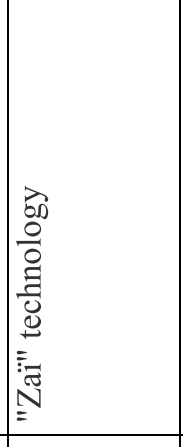 & 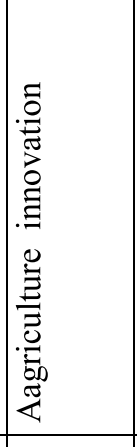 & 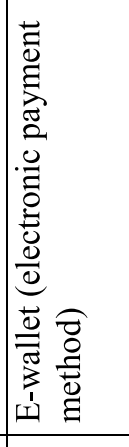 & 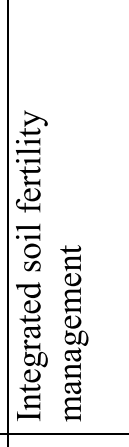 & 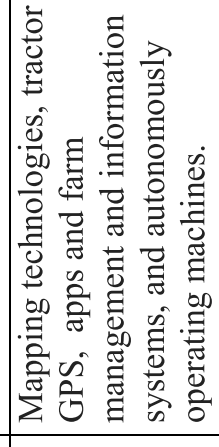 & 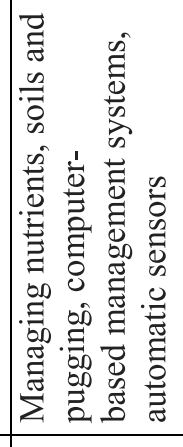 & 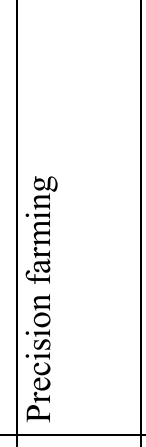 & 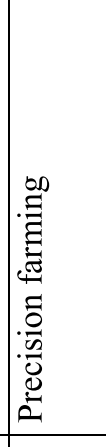 \\
\hline 吾 & 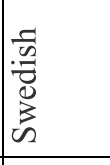 & 空 & 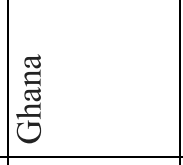 & 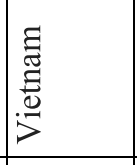 & ${ }_{\mathrm{s}}^{\mathrm{s}}$ & 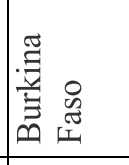 & 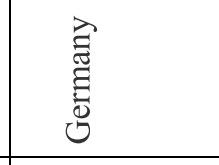 & 焉 & 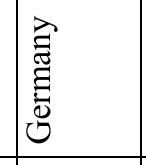 & 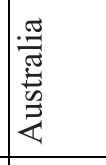 \\
\hline 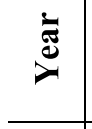 & तें & 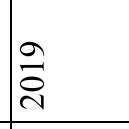 & 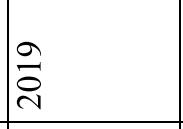 & $\sqrt{2}$ & సें & $\vec{\sim}$ & $\overrightarrow{\mathrm{i}}$ & $\bar{\sim}$ & $\overrightarrow{\stackrel{\sim}{\vec{\nu}}}$ & iे \\
\hline 竞 & 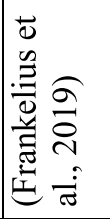 & 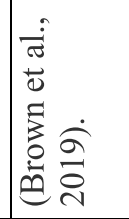 & 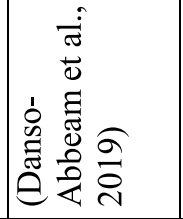 & 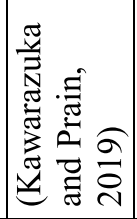 & & 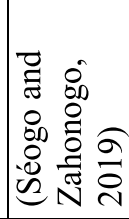 & 耐 & 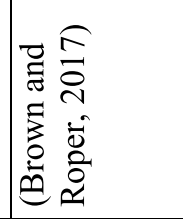 & 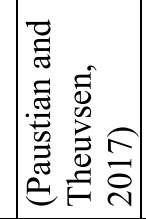 & 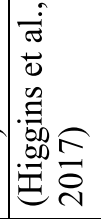 \\
\hline
\end{tabular}




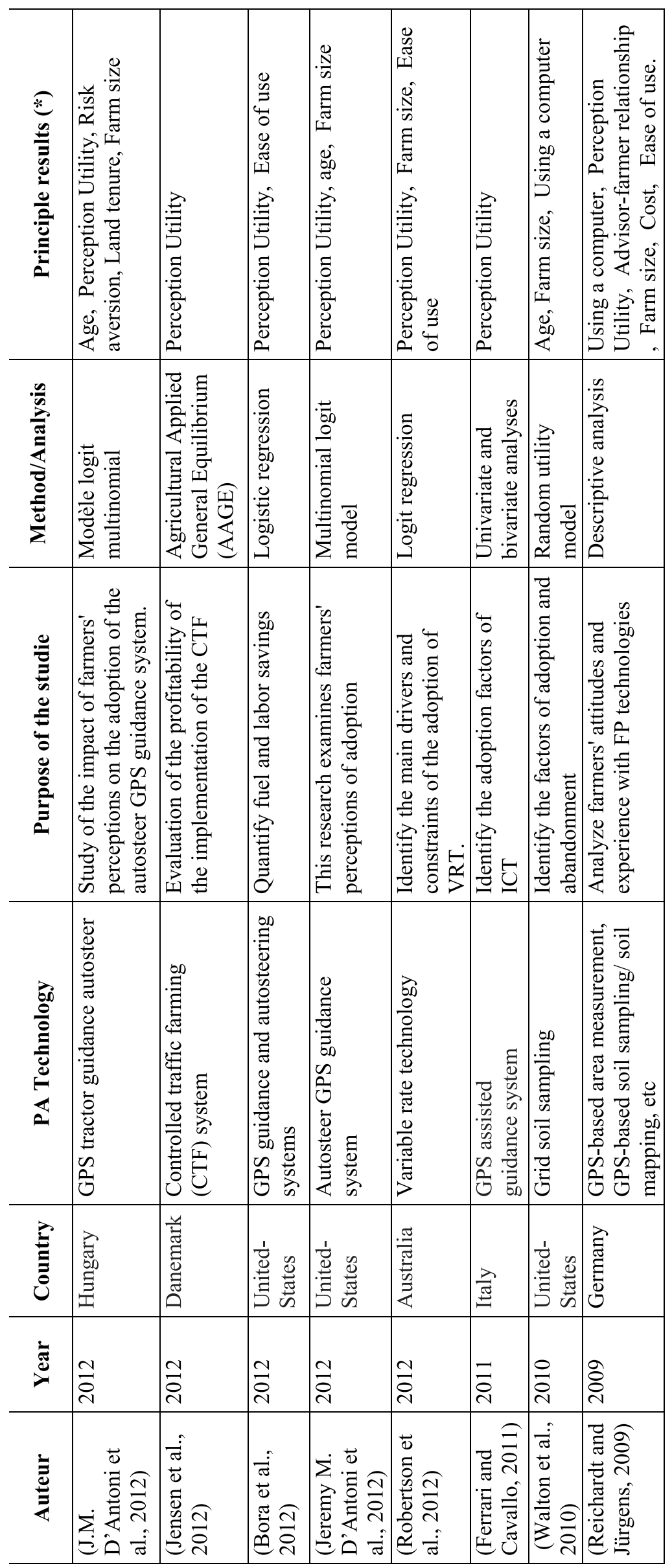




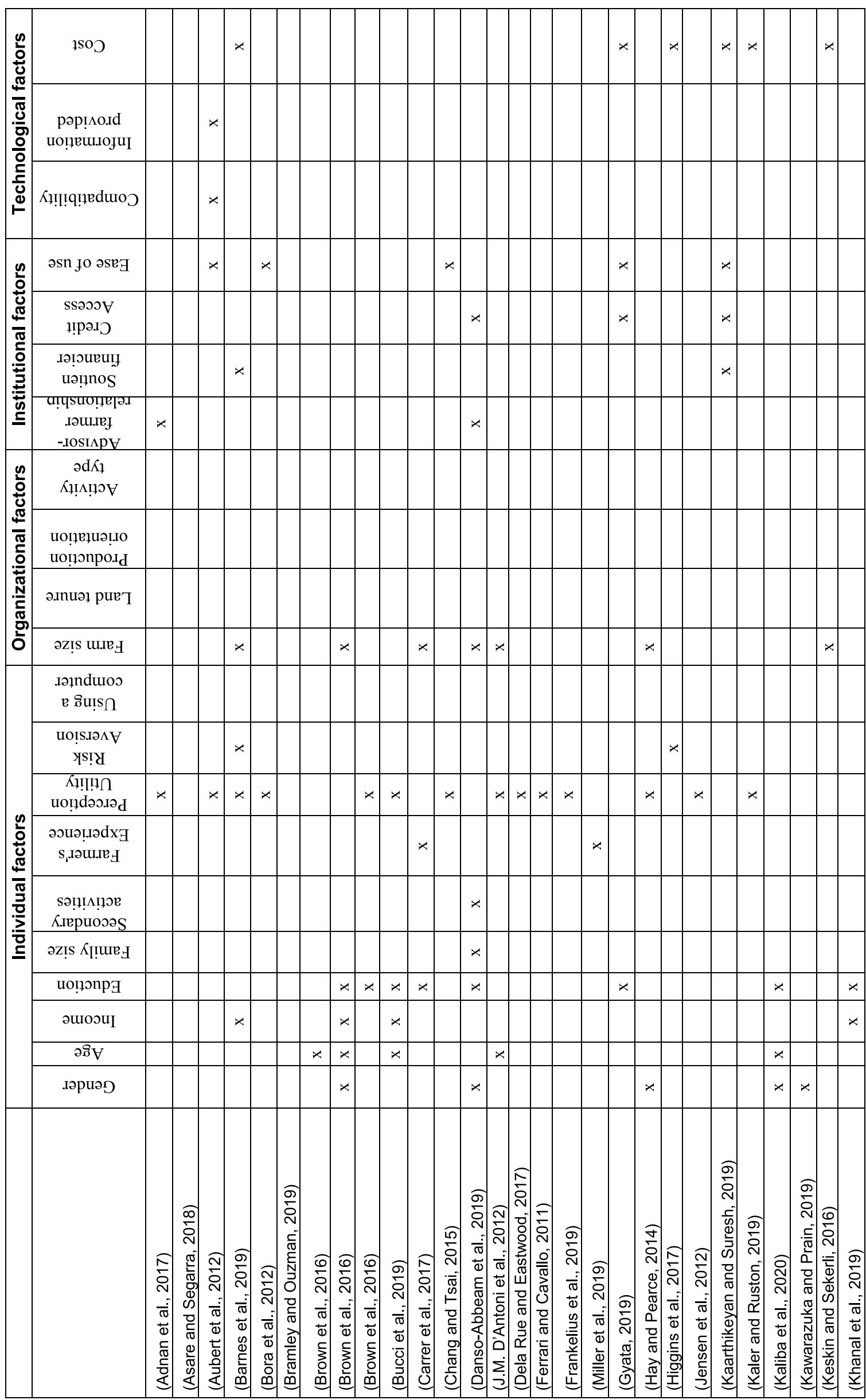



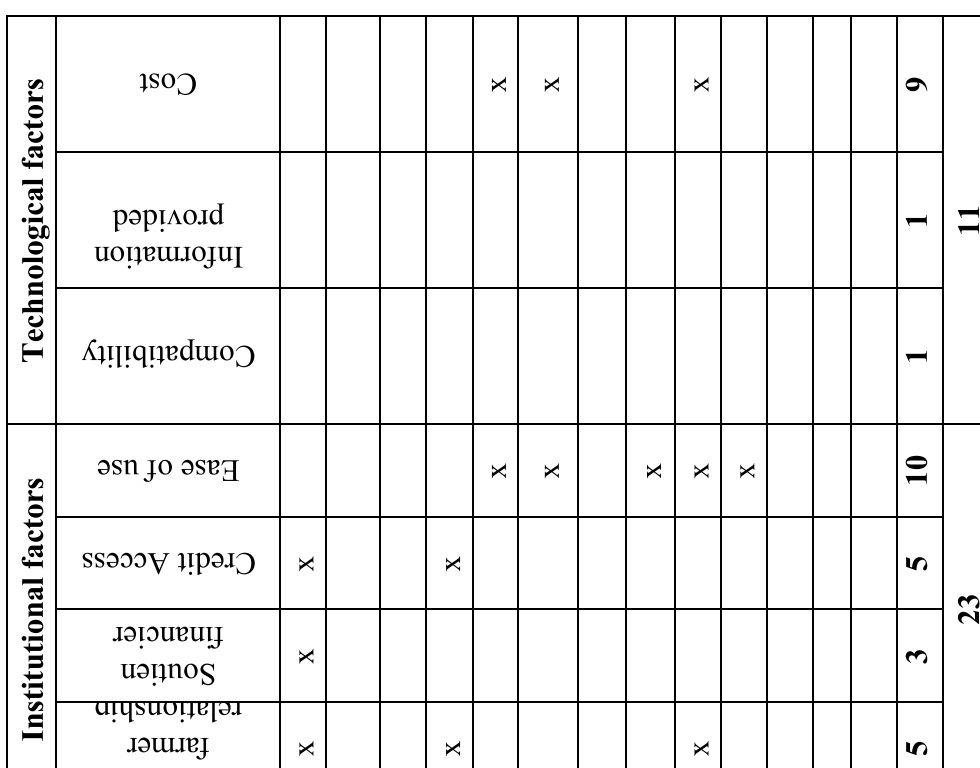

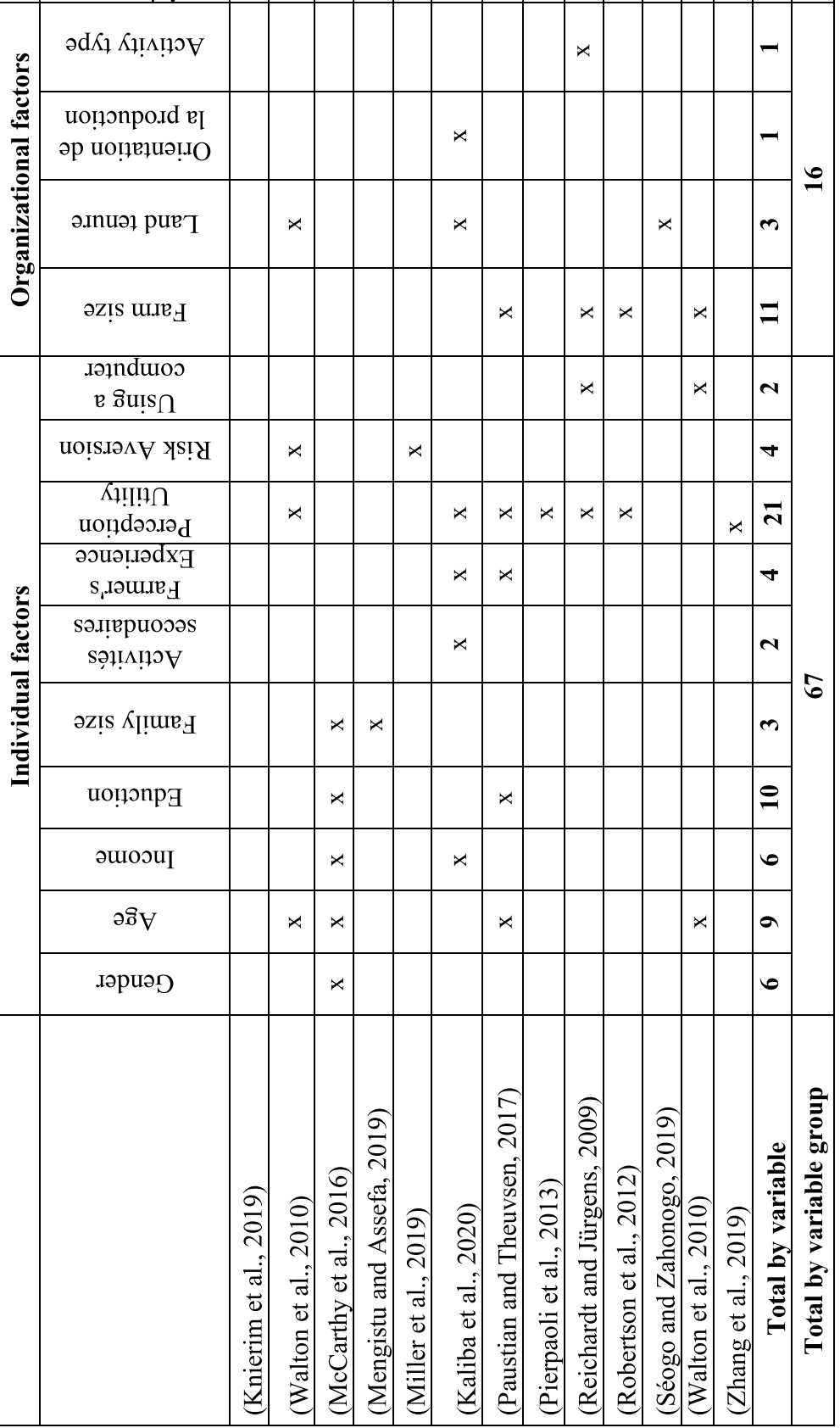


Table 4. Degree of importance of the factors according to the number of times identified

\begin{tabular}{|c|c|c|c|}
\hline $\begin{array}{l}\text { Importance } \\
\text { Degree }\end{array}$ & Factors & $\begin{array}{l}\text { Number times } \\
\text { identified }\end{array}$ & $\begin{array}{l}\text { Identification } \\
\text { Frequency (\%) }\end{array}$ \\
\hline \multirow{4}{*}{$\begin{array}{c}\text { Level } 1 \\
\text { (High) }\end{array}$} & Perception Utility & 21 & 5,3 \\
\hline & Farm size & 11 & 3,1 \\
\hline & Education & 10 & 2,8 \\
\hline & Ease of use & 10 & 2,8 \\
\hline \multirow{8}{*}{$\begin{array}{c}\text { Level } 2 \\
\text { (Way) }\end{array}$} & Age & 9 & 2,5 \\
\hline & Cost & 9 & 2,5 \\
\hline & Gender & 6 & 1,7 \\
\hline & Income & 6 & 1,7 \\
\hline & $\begin{array}{l}\text { Farmer-consultant } \\
\text { relationship }\end{array}$ & 5 & 1,4 \\
\hline & Credit Access & 5 & 1,4 \\
\hline & Farmer's Experience & 4 & 1,1 \\
\hline & Risk Aversion & 4 & 1,1 \\
\hline \multirow{9}{*}{$\begin{array}{c}\text { Level } 3 \\
\text { (Low) }\end{array}$} & Family size & 3 & 0,8 \\
\hline & Land tenure & 3 & 0,8 \\
\hline & Financial support & 3 & 0,8 \\
\hline & Secondary activity & 2 & 0,6 \\
\hline & Using a computer & 2 & 0,6 \\
\hline & $\begin{array}{l}\text { Orientation de la } \\
\text { production }\end{array}$ & 1 & 0,3 \\
\hline & Activity type & 1 & 0,3 \\
\hline & Compatibility & 1 & 0,3 \\
\hline & Information provided & 1 & 0,3 \\
\hline
\end{tabular}

\subsection{INDIVIDUAL FACTORS}

Age is identified four times as determinants of adoption on the 41 articles selected. Older farmers tend to be conservative and reluctant to change and adopt technologies due to risk aversion (Kaliba et al., 2020). Brown et al., (2019) explains that older farmers are more risk-averse, less motivated to experiment with new technologies, less likely to be influenced by the benefits of new technologies. However, Paustian and Theuvsen (2017) explains that young farmers are more open to innovations and more likely to adopt new technologies. Reichardt and Jürgens (2009) add that sometimes the adoption of technologies by older farmers is explained by the high investment costs, and that these farmers already have the means to acquire them, unlike the younger ones.

Education is ranked as the third most identified factor with 11 times identified as a determinant of adoption. The level of education is emphasized by many authors as a determinant of the adoption of new technologies (Bucci and al., 2019; Carrer and al., 2017; Gyata, 2019; Reichardt and Jürgens, 2009). For example, (Kaliba et al., 2020), find that the adoption rate increases with the level of education of the farmer, especially if technology is advanced, and that learning is necessary for its use. farmer its use, but also, becomes someone more open to innovation.

The gender factor is also a determinant of adoption (6 times identified). However, the influence of gender on the adoption of new technologies remains unclear. While (Danso-Abbeam et al., 2019) shows that men are more likely to adopt technology than women, (Hay and Pearce, 2014), finds 
that women have a more positive perception than men about the benefits of new technologies, it highlights the interest of rural women in technology which has enabled them to derive substantial benefits, for example by adopting a livestock management system. The author adds that rural women use three times more new technologies than men.

As mentioned above, the cost of acquisition has been closely linked in the literature to agricultural and non-agricultural income (6 times identified). Research shows that the higher the farm income, the more likely the farmer to adopt new technologies (Miller et al., 2019). The existence of nonfarm income also increases the chances of adoption (Ng'ang'a et al., 2019). Barnes et al. (2019) explains that for farmers with moderate income, off-farm income provides cash that can help the farmer acquire new technologies. Access to off-farm income-generating activities is generally associated with the adoption of technology. Griffin et al. (2017) found that adoption has high entry costs and that higher income farmers are more likely to adopt it. Lambert et al. (2015) showed that a $10 \%$ increase in income from farming was associated with a $9.2 \%$ increase in the chances that a producer would adopt these technologies.

Perceived utility tops the ranking with twenty-one times identified as a determinant of adoption. Farmers are more likely to adopt new technologies after seeing their usefulness in the field (Barnes and al., 2019; Brown and Roper, 2017; D’Antoni and al., 2012; Dela Rue and Eastwood, 2017; Griffin and al., 2017; Mengistu and Assefa, 2019, 2019; Ng'ang'a and al., 2019). For example, Brown and Roper (2017), in a study on the adoption of technologies in the dairy sector in Italy, he indicates that the demonstration of new technologies are practically done within networks of farmers, which push them thereafter to adopt the same technology (Brown and Roper, 2017). Zhang et al. (2019) adds that cleaner production techniques (CPT) will not be adopted only when the farmers have perceived their usefulness, but also, have seen the satisfaction of other farmers who have already adopted this technology.

On the other hand, several authors consider that the farmer's experience is an indicator of his skills in the farming profession (Brown and al., 2019; Carrer and al., 2017; Griffin and al., 2017; Paustian and Theuvsen, 2017). The experience factor is identified as a determinant of adoption, but the research results do not lead to the same conclusions. While Paustian and Theuvsen (2017) showed that an experience of less than 5 years, and between 16 and 20 years in agriculture promotes the adoption of new technologies, Carrer et al. (2017) found that 1 farmers' experience has a negative impact on the adoption of information systems.

A review of the literature reveals another important factor affecting the adoption of new technologies, namely the attitude to risk. Some farmers prefer to work with the means at their disposal rather than investing in new technologies (Miller and al., 2019). This reluctance can be explained in particular by the uncertainty, first of all about the use of technology, and then about economic return. For example, when the market price of agricultural products falls, farmers tend to invest less in capital (machinery, innovation, etc.). For example, Barnes et al. (2019) points out that farmers who have less uncertainty about the economic return of VRNT are more likely to invest in these technologies. Complex technologies require additional investment in learning. Ng'ang'a and al. (2019) adds that a low level of skills and know-how have a negative influence on adoption. Its results show that technologies that require specific skills and technical know-how decrease the likelihood of their adoption.

Danso-Abbeam and al. (2019) find that household size explains the probability and intensity of adoption. This could be attributed to the availability of labor that is none other than household members. Kaliba and al. (2020) explains that labor availability is a crucial factor in technology adoption, especially when labor supply is scarce, and it is difficult to hire additional labor. Otherwise, adoption of labor-intensive technologies is likely if the labor force is plentiful and cheap, or the chances of household members finding non-farm employment are reduced. In the same perspective, Ng'ang'a and al. (2019) reported a negative relationship between the cost of labor and the likelihood of adopting labor-intensive farming practices. 
Finally, the use of computers in farm management has been associated with the adoption of certain technology, such as a PDA (Walton and al., 2010), or the autosteer GPS guidance system (D'Antoni and al., 2012). Walton and al. (2010) explains that farmers who use computers in administrative management (for mail, invoices, for accounting, inventory management, human resource management, etc.) may be more likely to adopt the technology of 'AP. Reichardt and Jürgens (2009) postulate that farmers who are open-minded about technological progress are also more favorable to the adoption of technologies on their farms.

\section{ORGANIZATIONAL FACTORS}

Farm size comes second with 11 times identified as a determinant of adoption. Carrer et al. (2017) explains that large farms are more complex to manage, new technologies have proven to be effective in optimizing production and reducing costs. Danso-Abbeam and al., (2019) explains that the size of the farm is linked to the adoption of new technologies, because the farmer tends to devote part of his land to trying out a new technology first, unlike farms short. These results align with several results from previous studies on the adoption of PA technologies (Brown and al., 2019; Carrer et al., 2017; Paustian and Theuvsen, 2017; Reichardt and Jürgens, 2009; Welsh and al., 2010). The cost of acquiring technology is often identified as a determinant of adoption ( 9 times identified) (Carrer and al., 2017; Chang and Tsai, 2015; Keskin and Sekerli, 2016; Khanal and al., 2019). Gyata (2019) and Reichardt and Jürgens (2009) points out that the majority of farmers are reluctant to introduce precision farming techniques mainly due to their high costs. Large farms are more likely to adopt precision farming technologies by small farms because of their financial capacity (Barnes and al., 2019). However, the cost of acquisition has been closely linked in the literature to agricultural and non-agricultural income.

On the other hand, Barnes and al. (2019) points out that the lack of reduced land tenure would target the answer for farmers to adopt the technologies. Indeed, farmers with title deeds are more likely to have practices that improve the soil compared to those without title deeds. This result is similar to that of (Barnes and al., 2019), indicating that the land title gives farmers their rights to use the land. Studies (Lambert and al., 2015; Séogo and Zahonogo, 2019; Welsh and al., 2010) have indicated that farmers who have obtained property certificates feel more secure, which allows them to make the long-term investment. This is the case for example of drip, its non-adoption is most often linked to the location status which does not benefit from subsidies.

On the other hand, some studies have shown that farmers who practice commercial farming are more likely to see the technologies, as they often seek to improve their production compared to farmers who practice subsistence farming. (Barnes et al., 2019) finds that the results obtained from the technologies increase by $10 \%$ for commercial farmers, but decrease by $26 \%$ for subsistence farming. The author explains that this could also be due to the fact that commercial farmers are risk takers compared to those who are not commercial.

As for the activity type, Lambert and al. (2015), showed that the adoption process is a dynamic and complex process, linked to changes in land use. For example, livestock has a positive effect on the process of adopting new technologies. In addition, Lambert et al. (2015) showed that the factors that influence the timing of adoption are linked to the characteristics of the farm. Thus, (Adnan et al., 2017; Mengistu and Assefa, 2019) have shown that the variable "crops grown" on the other hand has a determining effect. Many farmers argue that drip is not suitable for old plantations, because they say this technique does not meet the water requirements of trees that have already developed a deep root system. On the other hand, market gardeners in greenhouses are often adepts of drip. 


\section{INSTITUTIONAL FACTORS}

Most studies show the importance of public service intervention in relation to the adoption of new technologies. It has been shown that awareness (Adnan and al., 2017; Mengistu and Assefa, 2019), access to agricultural extension services (Mengistu, 2019), credit facilities and the practice of aggregation (Barnes et al., 2019; Danso-Abbeam and al., 2019; Gyata, 2019; Kaarthikeyan and Suresh, 2019), are essential to encourage the adoption of technological innovations.

Mengistu and Assefa (2019) finds that training and extension are means of raising awareness and supporting the adoption of watershed management practices. Reichardt and Jürgens (2009) emphasizes the importance of a good advisory service, and of providing the necessary information. The author points out that lack of counseling and training are barriers to adoption, and that the availability of the internet alone is not a sufficient factor for farmers.

Farmers' access to credit services is identified as a determinant of the intensity of adoption, because bank credit allows farmers to have other financial resources, and therefore invest in new technologies. Several studies (Danso-Abbeam and al., 2019; Gyata, 2019; Kaarthikeyan and Suresh, 2019) conclude that farmers' access to credit services increases the adoption of technologies.

Barnes and al. (2019) have shown that subsidies and taxation are considered to be positive drivers for the adoption of new technologies. Adnan and al. (2017) indicated that the adoption of green fertilizers is strongly linked to certain financial initiatives, such as capital grants for maintenance, reduction of the tax for adopters, reduction of the interest rate and technical supplements for the adoption of green fertilizers in order to reduce costs and therefore increase yields.

Kaarthikeyan shows that the variable «amount of subsidy» is strongly correlated with the adoption rate, the higher this amount, the greater the probability of adoption. Logically, the variable «difficulty in accessing the subsidy» is correlated with a relatively low rate of adoption. The more difficult farmers find it to access the subsidy, the lower the probability that they will adopt drip.

\section{TECHNOLOGICAL FACTORS}

Ease of use is identified as an important factor in the adoption of PA technologies (10 times identified). Adnan and al. (2017) finds that farmers' intention to adopt AMPs is influenced by perceived ease of use, subjective standards and perceived behavioral control. Analysis shows that farmers' perception of the ease of use of PA technology has a significant impact on its adoption. A farmer who perceives PA technology as complex or difficult to use is therefore less likely to adopt it (Aubert and al., 2012).

On the other hand, the issue of technology compatibility was often raised when studying perceived utility. Aubert and al. (2012) emphasize the importance of technology compatibility, it shows that "technology compatibility" has had the strongest influence on the perceived usefulness of the technology and a very strong influence on the ease of use. Lack of compatibility can create uncertainty and reluctance on the part of farmers to spend large capital expenditures on PA technology (Higgins and al., 2017). Therefore, setting standards that match farmers' practices would maximize compatibility, which in turn would increase both perceived usefulness and ease of use, and ultimately increase adoption (Aubert et al., 2012). Finally, Nordin et al.(2014) found that the adoption process requires several variables, such as the quality of the information provided by the technology. 


\section{CONCLUSION}

The purpose of this contribution was to synthesize the last ten years of literature on the adoption of precision farming technologies. Using the prisma statement method (Preferred Reporting Items for Systematic reviews and Meta-Analyzes), 41 articles were selected in this systematic review. An in-depth analysis was conducted to identify all of the factors that impact farmers' adoption of the technologies. The main elements found were listed in Tables 2, 3 and 4. The results of this systematic review, as well as the limits and perspectives of research can be summarized as follows: The results first show that individual factors are most identified as determinants of the adoption of precision farming technologies. This means that the farmer is at the center of the adoption decision. On the other hand, perceived utility remains the factor most identified in this systematic literature as a determinant of adoption. Thus, according to the results obtained, the impact of certain factors on adoption is not unanimous. The farmer's gender and experience are not always determinants. More work is still needed at this level.

In most studies, adoption factors were treated separately. Analysis of the results reveals that there are direct relationships between the factors themselves (for example, income with the cost of acquiring technology are closely linked). This suggests that in future work, it would be more relevant to treat these factors as covariates or moderating variables.

This study is not without limits. First, there is likely unpublished work and research in languages other than English that was not considered and included in this study. English being the main language for research dissemination in the world, and given that it is generally difficult to have access to unpublished documents, these limits are not likely to strongly affect the results obtained. Then, the heterogeneity of the included studies does not allow to make comparisons or metaanalyzes or draw conclusions for future applications. This implies that this area still needs further research. It also appeared that the literature dealing with the determinants comes from Englishspeaking countries also creating a selection bias. Questions arise at this level, would the nature of the determinants of adoption depend on the level of development of the countries? It is reasonable to assume that farmers in a developed country are more influenced by individual factors than by institutional factors. For example, in developing countries, where institutional blockages (such as red tape for the use of drones, lack of financial support and advice) are more answered. You might even think that the spirit of innovation is more developed in some countries than others. At this level, a research avenue which seems interesting, namely: the evaluation of the spirit of innovation among farmers in relation to the adoption of new agricultural technologies. According to the results of this research, no study has been interested in this aspect. Ultimately, this work can form the basis for further empirical work on the determinants of adoption. It can also serve the public authorities to set up a policy for the modernization of the agricultural sector. Digitization has today become an imperative in all sectors of the economy, including agriculture. COVID-19 will certainly accelerate this desire for modernization, but its success depends first of all on an understanding of the mechanisms that lead the farmer to the acceptance and adoption of new technologies. 


\section{REFERENCES}

Adnan, N., Nordin, S.M., bin Abu Bakar, Z., 2017. Understanding and facilitating sustainable agricultural practice: A comprehensive analysis of adoption behaviour among Malaysian paddy farmers. Land Use Policy 68, 372-382. https://doi.org/10.1016/j.landusepol.2017.07.046

Asare, E., Segarra, E., 2018. Adoption and extent of adoption of georeferenced grid soil sampling technology by cotton producers in the southern US. Precis. Agric. 19, 992-1010. https://doi. org/10.1007/s11119-018-9568-3

Aubert, B.A., Schroeder, A., Grimaudo, J., 2012. IT as enabler of sustainable farming: An empirical analysis of farmers' adoption decision of precision agriculture technology. Decis. Support Syst. 54, 510-520. https://doi.org/10.1016/j.dss.2012.07.002

Bagheri, N., Bordbar, M., 2014. Solutions for fast development of precision agriculture in Iran. Agric. Eng. Int. CIGR J. 16, 119-123.

Barnes, A.P., Soto, I., Eory, V., Beck, B., Balafoutis, A., Sánchez, B., Vangeyte, J., Fountas, S., van der Wal, T., Gómez-Barbero, M., 2019. Exploring the adoption of precision agricultural technologies: A cross regional study of EU farmers. Land Use Policy 80, 163-174. https://doi. org/10.1016/j.landusepol.2018.10.004

Bora, G.C., Nowatzki, J.F., Roberts, D.C., 2012. Energy savings by adopting precision agriculture in rural USA. Energy Sustain. Soc. 2, 1-5. https://doi.org/10.1186/2192-0567-2-22

Bramley, R.G.V., Ouzman, J., 2019. Farmer attitudes to the use of sensors and automation in fertilizer decision-making: nitrogen fertilization in the Australian grains sector. Precis. Agric. 20, 157175. https://doi.org/10.1007/s11119-018-9589-y

Brown, P., Daigneault, A., Dawson, J., 2019. Age, values, farming objectives, past management decisions, and future intentions in New Zealand agriculture. J. Environ. Manage. 231, 110120. https://doi.org/10.1016/j.jenvman.2018.10.018

Brown, P., Hart, G., Small, B., de Oca Munguia, O.M., 2016. Agents for diffusion of agricultural innovations for environmental outcomes. Land Use Policy 55, 318-326. https://doi. org/10.1016/j.landusepol.2016.04.017

Brown, P., Roper, S., 2017. Innovation and networks in New Zealand farming. Aust. J. Agric. Resour. Econ. 61, 422-442. https://doi.org/10.1111/1467-8489.12211

Bucci, G., Bentivoglio, D., Finco, A., 2019. Factors affecting ict adoption in agriculture: A case study in italy. Qual. - Access Success 20, 122-129.

Carrer, M.J., de Souza Filho, H.M., Batalha, M.O., 2017. Factors influencing the adoption of Farm Management Information Systems (FMIS) by Brazilian citrus farmers. Comput. Electron. Agric. 138, 11-19. https://doi.org/10.1016/j.compag.2017.04.004

Chang, S.C., Tsai, C.-H., 2015. The adoption of new technology by the farmers in Taiwan. Appl. Econ. 47, 3817-3824. https://doi.org/10.1080/00036846.2015.1019035

Danso-Abbeam, G., Dagunga, G., Ehiakpor, D.S., 2019. Adoption of Zai technology for soil fertility management: evidence from Upper East region, Ghana. J. Econ. Struct. 8. https://doi. org/10.1186/s40008-019-0163-1

D’Antoni, J.M., Mishra, A.K., Joo, H., 2012. Farmers' perception of precision technology: The case of autosteer adoption by cotton farmers. Comput. Electron. Agric. 87, 121-128. https://doi. org/10.1016/j.compag.2012.05.017

D’Antoni, Jeremy M., Mishra, A.K., Joo, H., 2012. Farmers' perception of precision technology: The case of autosteer adoption by cotton farmers. Comput. Electron. Agric. 87, 121-128. https:// doi.org/10.1016/j.compag.2012.05.017

Dela Rue, B.T., Eastwood, C.R., 2017. Individualised feeding of concentrate supplement in pasturebased dairy systems: Practices and perceptions of New Zealand dairy farmers and their advisors. Anim. Prod. Sci. 57, 1543-1549. https://doi.org/10.1071/AN16471 
Ferrari, E., Cavallo, E., 2011. Issues in new technology adoption in agriculture: A survey among italian tractor's users. Presented at the CEUR Workshop Proceedings, pp. 121-128.

Frankelius, P., Norrman, C., Johansen, K., 2019. Agricultural Innovation and the Role of Institutions: Lessons from the Game of Drones. J. Agric. Environ. Ethics 32, 681-707. https://doi. org/10.1007/s10806-017-9703-6

Griffin, T.W., Miller, N.J., Bergtold, J., Shanoyan, A., Sharda, A., Ciampitti, I.A., 2017. Farm's sequence of adoption of information-intensive precision agricultural technology. Appl. Eng. Agric. 33, 521-527. https://doi.org/10.13031/aea.12228

Gyata, B.A., 2019. Comparative assessment of adoption determinants of electronic wallet system by rice farmers in Benue and Taraba states, Nigeria. Food Res. 3, 117-122. https://doi. org/10.26656/fr.2017.3(2).132

Hay, R., Pearce, P., 2014. Technology adoption by rural women in Queensland, Australia: Women driving technology from the homestead for the paddock. J. Rural Stud. 36, 318-327. https:// doi.org/10.1016/j.jrurstud.2014.10.002

Higgins, V., Bryant, M., Howell, A., Battersby, J., 2017. Ordering adoption: Materiality, knowledge and farmer engagement with precision agriculture technologies. J. Rural Stud. 55, 193-202. https://doi.org/10.1016/j.jrurstud.2017.08.011

Jensen, H.G., Jacobsen, L.-B., Pedersen, S.M., Tavella, E., 2012. Socioeconomic impact of widespread adoption of precision farming and controlled traffic systems in Denmark. Precis. Agric. 13, 661-677. https://doi.org/10.1007/s11119-012-9276-3

Kaarthikeyan, G.M., Suresh, A., 2019. A study on understanding the adoption of water saving technology: A case study of drip irrigation. Int. J. Recent Technol. Eng. 7, 1123-1130.

Kaler, J., Ruston, A., 2019. Technology adoption on farms: Using Normalisation Process Theory to understand sheep farmers' attitudes and behaviours in relation to using precision technology in flock management. Prev. Vet. Med. 170. https://doi.org/10.1016/j.prevetmed.2019.104715

Kaliba, A.R., Mushi, R.J., Gongwe, A.G., Mazvimavi, K., 2020. A typology of adopters and nonadopters of improved sorghum seeds in Tanzania: A deep learning neural network approach. World Dev. 127. https://doi.org/10.1016/j.worlddev.2019.104839

Kawarazuka, N., Prain, G., 2019. Gendered processes of agricultural innovation in the Northern uplands of Vietnam. Int. J. Gend. Entrep. 11, 210-226. https://doi.org/10.1108/IJGE-042019-0087

Keskin, M., Sekerli, Y.E., 2016. Awareness and adoption of precision agriculture in the Cukurova region of Turkey. Agron. Res. 14, 1307-1320.

Khanal, A.R., Mishra, A.K., Lambert, D.M., Paudel, K.K., 2019. Modeling post adoption decision in precision agriculture: A Bayesian approach. Comput. Electron. Agric. 162, 466-474. https:// doi.org/10.1016/j.compag.2019.04.025

Knierim, A., Kernecker, M., Erdle, K., Kraus, T., Borges, F., Wurbs, A., 2019. Smart farming technology innovations - Insights and reflections from the German Smart-AKIS hub. NJAS Wagening. J. Life Sci. 90-91. https://doi.org/10.1016/j.njas.2019.100314

Koutsos, T., Menexes, G., 2019. Economic, agronomic, and environmental benefits from the adoption of precision agriculture technologies: A systematic review. Int. J. Agric. Environ. Inf. Syst. 10, 40-56. https://doi.org/10.4018/IJAEIS.2019010103

Lambert, D.M., Paudel, K.P., Larson, J.A., 2015. Bundled adoption of precision agriculture technologies by cotton producers. J. Agric. Resour. Econ. 40, 325-345.

McCarthy, B., Liu, H.-B., Chen, T., 2016. Innovations in the agro-food system: Adoption of certified organic food and green food by Chinese consumers. Br. Food J. 118, 1334-1349. https://doi. org/10.1108/BFJ-10-2015-0375

Mengistu, F., Assefa, E., 2019. Farmers' decision to adopt watershed management practices in Gibe 
basin, southwest Ethiopia. Int. Soil Water Conserv. Res. 7, 376-387. https://doi.org/10.1016/j. iswcr.2019.08.006

Miller, N.J., Griffin, T.W., Ciampitti, I.A., Sharda, A., 2019. Farm adoption of embodied knowledge and information intensive precision agriculture technology bundles. Precis. Agric. 20, 348361.

Ng'ang'a, S.K., Jalang'o, D.A., Girvetz, E.H., 2019. Adoption of technologies that enhance soil carbon sequestration in East Africa. What influence farmers' decision? Int. Soil Water Conserv. Res. https://doi.org/10.1016/j.iswcr.2019.11.001

Nordin, S.M., Noor, S.M., Saad, M.S. bin M., 2014. Innovation Diffusion of New Technologies in the Malaysian Paddy Fertilizer Industry. 2nd World Conf. Bus. Econ. Manag. 109, 768-778.

Paustian, M., Theuvsen, L., 2017. Adoption of precision agriculture technologies by German crop farmers. Precis. Agric. 18, 701-716. https://doi.org/10.1007/s11119-016-9482-5

Pierpaoli, E., Carli, G., Pignatti, E., Canavari, M., 2013. Drivers of Precision Agriculture Technologies Adoption: A Literature Review. 6th Int. Conf. Inf. Commun. Technol. Agric. Food Environ. HAICTA 2013 8, 61-69. https://doi.org/10.1016/j.protcy.2013.11.010

Reichardt, M., Jürgens, C., 2009. Adoption and future perspective of precision farming in Germany: Results of several surveys among different agricultural target groups. Precis. Agric. 10, 73-94. https://doi.org/10.1007/s11119-008-9101-1

Robertson, M.J., Llewellyn, R.S., Mandel, R., Lawes, R., Bramley, R.G.V., Swift, L., Metz, N., O'Callaghan, C., 2012. Adoption of variable rate fertiliser application in the Australian grains industry: Status, issues and prospects. Precis. Agric. 13, 181-199. https://doi.org/10.1007/ s11119-011-9236-3

Séogo, W., Zahonogo, P., 2019. Land tenure system innovation and agricultural technology adoption in Burkina Faso: Comparing empirical evidence to the worsening situation of both rural people vulnerability and vulnerable groups' access to land. Afr. J. Sci. Technol. Innov. Dev. 11, 833-842. https://doi.org/10.1080/20421338.2019.1587257

Walton, J.C., Roberts, R.K., Lambert, D.M., Larson, J.A., English, B.C., Larkin, S.L., Martin, S.W., Marra, M.C., Paxton, K.W., Reeves, J.M., 2010. Grid soil sampling adoption and abandonment in cotton production. Precis. Agric. 11, 135-147. https://doi.org/10.1007/s11119-009-9144-y

Watcharaanantapong, P., Roberts, R.K., Lambert, D.M., Larson, J.A., Velandia, M., English, B.C., Rejesus, R.M., Wang, C., 2014. Timing of precision agriculture technology adoption in US cotton production. Precis. Agric. 15, 427-446. https://doi.org/10.1007/s11119-013-9338-1

Welsh, R., Grimberg, S., Gillespie, G.W., Swindal, M., 2010. Technoscience, anaerobic digester technology and the dairy industry: Factors influencing north country new york dairy farmer views on alternative energy technology. Renew. Agric. Food Syst. 25, 170-180. https://doi. org/10.1017/S174217051000013X

Zhang, T., Yang, Y., Ni, J., Xie, D., 2019. Adoption behavior of cleaner production techniques to control agricultural non-point source pollution: A case study in the Three Gorges Reservoir Area. J. Clean. Prod. 223, 897-906. https://doi.org/10.1016/j.jclepro.2019.03.194 INTRODUCTION 